\title{
APRENDIZAJE MOTOR Y REALIMENTACIÓN: CONSIDERACIONES PRÁCTICAS
}

\author{
MOTOR LEARNING AND FEEDBACK: PRACTICAL CONSIDERATIONS
}

\author{
Óscar Alfredo Montenegro Arjona ${ }^{1}$
}

\section{Resumen}

\begin{abstract}
En el presente artículo se aborda el tema del aprendizaje motor y se hace énfasis en la realimentación como un aspecto relevante. Inicialmente, se hace una contextualización de su importancia en el proceso de aprendizaje; luego, se exponen las diferentes clases de realimentación que puede recibir un practicante, en particular, la de tipo extrínseco. Enseguida, se esbozan varias consideraciones prácticas a tener en cuenta en el momento de brindar realimentación en diferentes contextos del aprendizaje motor. Para finalizar, se proponen algunas ideas a manera de conclusiones.
\end{abstract}

Palabras claves: aprendizaje motor, realimentación intrínseca, realimentación extrínseca.

\section{Abstract}

This article tackles motor learning with emphasis in feedback as a vital aspect in the process of learning. Initially, there is a contextualization of the importance of feedback in the process of learning; then, the author exposes different kinds of feedback that a learner can receive making emphasis in the extrinsic feedback. Afterward, the author explains some practical considerations to take into a count at the moment to give feedback in different context of motor learning. Finally, some ideas are proposed as conclusions.

Keywords: motor learning, intrinsic feedback, extrinsic feedback.

Fecha de recepción: 15 de mayo de 2015

Fecha de aprobación: 10 de octubre de 2015

Para citar este artículo:

Montenegro, O.A. (2015). Aprendizaje motor y realimentación, consideraciones prácticas. Lúdica Pedagógica, (22), 75-83.

1 Magíster en Pedagogía de la Cultura Física, Licenciado en Educación Física. Profesor de Tiempo Completo, Universidad Surcolombiana. Tutor del Semillero de Investigación Sprung. Grupo de Investigación Altius. Correo electrónico: alfredo.montenegro@usco.edu.co 


\section{INTRODUCCIÓN}

El aprendizaje motor es explicado por algunos investigadores a partir de un modelo teórico que interpreta al practicante (aprendiz) como un procesador de información (Schmidt, 2003; Maguire, 2014; Shea y Wulf, 2005). En esta propuesta, la persona inicia interactuando con la información disponible del ambiente; luego, la misma y, por último, produce una respuesta acorde al estímulo inicialmente percibido.

Durante el procesamiento de la información, las actividades de percepción, memoria, intensión y acción, deben ser entendidas como una autoorganización formada por muchos componentes del sistema e importantes estímulos del ambiente (Davids, Button y Bennett, 2008). Por ejemplo, los componentes del sistema pueden ser neuronas del cerebro disparando simultáneamente estímulos para formar ideas, memoria y acciones; o pueden envolver grupos de músculos que abarcan varias articulaciones para formar movimientos coordinados. Los estímulos del ambiente son las informaciones percibidas a través de algunos sentidos importantes como la visión, la audición, el equilibrio o la propiocepción cinestésica. Pero, ¿cómo puede el practicante generar movimientos coordinados o manejar la información percibida para la acción motriz?

La información motriz perceptual, importante para el aprendizaje motor, puede ser representada dentro del sistema nervioso central. Dichas representaciones son adquiridas a través del aprendizaje, cuyos resultados almacenan un grupo de comandos de movimiento que controlan el comportamiento motor, en el sistema nervioso central. En caso de ser necesarios, los comandos de movimiento se pueden solicitar y transmitir a los músculos esqueléticos en forma de órdenes para reproducir el movimiento deseado. En el aprendizaje motor, los comandos de movimiento son equivalentes a la adquisición de modelos de movimiento, mejor conocidos como programas motores. Un programa motor contiene los impulsos o comandos de movimiento correspondientes a lo que el practicante quiere hacer; son formaciones neuronales de modelos de transmisión de impulsos en el sistema nervioso central condicionados por actividades de percepción y aprendizaje (Fairbrother, 2010).

Se dice que por cada habilidad motriz que una persona desee realizar, debe haber un programa motor que permita su ejecución. Así, la capacidad para coordinar movimientos de acuerdo con el objetivo de la habilidad y la situación donde esta se ejecuta, es el resultado de procesos de aprendizaje que producen cambios estructurales en el sistema nervioso central. Los procesos de aprendizaje dejan huellas que permiten un incremento en los puntos de conexión de las neuronas y, por ende, en la forma como se transmite la información. Por ello, el resultado de aprender se denomina memoria, que cuando se refiere específicamente a las experiencias de movimiento, se habla de memoria motora, como lo referencian Martin, et al (2004) y Wilmore y Costill (2002).

La memorización duradera de las informaciones adquiridas por el aprendizaje, es una conexión interneuronal, en la que asociaciones de células nerviosas están conectadas mediante sinapsis, como lo mencionan Geinisman et al. (2001). Se parte de la idea de que las sinapsis participantes en la formación de un modelo de impulso son especialmente receptivas a las secuencias de estímulos (Ma et al., 2011). Es decir, para que el aprendizaje de habilidades (técnicas deportivas u otras habilidades complejas) logre la estabilización de un movimiento valorado como correcto y susceptible de ser memorizado, necesita que muchos intentos sean realizados para que el modelo de impulsos nerviosos se grabe mediante los cambios de las sinapsis participantes. De esta manera, el aprendizaje de una habilidad motriz se explica en principio como la adquisición de programas motores, donde la realimentación cumple un papel muy importante en la adquisición de programas fuertemente conformados.

La realimentación es considerada la vía más importante para el aprendizaje motor, exceptuando por supuesto la práctica misma, como lo mencionan Schmidt y Lee (2005). Así, muchos investigadores están de acuerdo en que la realimentación debe ser tenida en cuenta de manera especial en los procesos de enseñanza/ aprendizaje, pues su efecto es un aspecto esencial en el aprendizaje (Marschall, Bund, y Wiemeyer, 2007; Marques, Ribeiro, Santos y Arroyo, 2010; Fairbrother, 2010). En este sentido, la preocupación central del presente documento es exponer la importancia de la realimentación en el aprendizaje motor y en la integración de programas motores estables. 


\section{LA REALIMENTACIÓN COMO}

\section{UN ASPECTO ESENCIAL DEL APRENDIZAJE}

Las personas pueden recibir distinta clase de información sensorial relacionada con sus movimientos, la cual puede estar disponible antes, durante o después del movimiento (Schmidt y Lee, 2005). La información que surge después del movimiento es conocida como realimentación (Marques et al., 2010) y se refiere a cómo el resultado del movimiento fue producido en el ambiente o a cómo el practicante sintió o realizó el movimiento; aunque, de acuerdo con Schmidt y Wrisberg (2008), la realimentación es definida como cualquier clase de información sensorial producida como resultado de movimientos. Es de aclarar que según el Diccionario de la Lengua Española, vigésima tercera edición, la palabra retroalimentar no está registrada.

La realimentación se puede clasificar de dos formas, según la fuente de información. En este sentido y teniendo como referencia a Fairbrother (2010), Schmidt y Wrisberg (2008), Schmidt y Lee (2005) y Zelaznik (1996), se considera que hay realimentación intrínseca y realimentación extrínseca. La figura 1 expone las manifestaciones de la realimentación mencionadas.

Figura 1. Manifestaciones de la realimentación con sus receptores

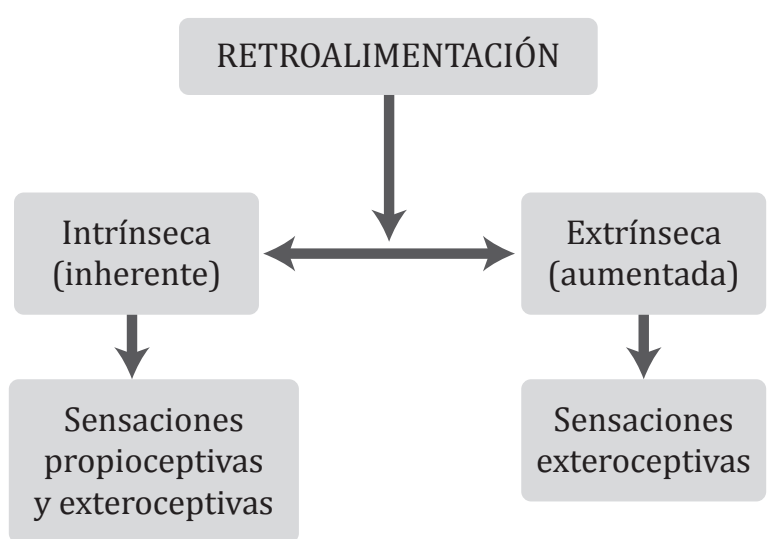

\section{LA REALIMENTACIÓN INTRÍNSECA}

La realimentación intrínseca o inherente al individuo es la información sensorial que ocurre cuando se produce un movimiento y el practicante es capaz de percibir la realimentación directamente por sí mismo, sin la especial asistencia de otras fuentes como: entrenador, juez, espectadores o algún objeto tecnológico como una cámara de video.
En la realimentación intrínseca, los encargados de recibir la información son los órganos de las sensaciones exteroceptivas y los de las sensaciones propioceptivas (Baechle y Earle, 2008; Haywood, y Getchell, 2009). Algunos ejemplos de la información captada intrínsecamente incluyen el sonido de la mano haciendo contacto con el balón de voleibol al momento de servir; la sensación de la cabeza, cuello y hombros al cabecear un balón de fútbol; la posición del hombro o el brazo en el momento de contacto de la raqueta con la bola de tenis, o la sensación general de un gimnasta haciendo el ejercicio de rondada o el flick flack.

\section{LA REALIMENTACIÓN EXTRÍNSECA}

La realimentación extrínseca, o aumentada, es la información sensorial que ocurre cuando se produce un movimiento y el practicante percibe la realimentación que proviene directamente de otras fuentes externas al cuerpo de la persona, como la que puede brindar el entrenador, un juez, los espectadores o algún objeto tecnológico como una cámara de video. En este sentido, la realimentación extrínseca, a diferencia de la intrínseca, es información suplementaria que aumenta la información de la realimentación inherente.

En la realimentación extrínseca, los encargados de recibir la información son los órganos de las sensaciones exteroceptivas (Clarky Russell, 2007). Algunos ejemplos de información captada extrínsecamente incluyen: el tiempo mostrado por un cronómetro a un corredor de 100 metros planos, los comentarios verbales que hace el profesor/entrenador a su practicante acerca de aspectos del proceso del movimiento ejecutado en alguna habilidad deportiva o la reproducción de un video mostrando a un futbolista en la ejecución de un tiro libre.

La extrínseca es el tipo de realimentación más importante a tener en cuenta en los procesos de enseñanza/ aprendizaje; pues los profesores o entrenadores son quienes controlan su dosificación. Por ello, para el desarrollo del tema, se abordará únicamente al estudio de la realimentación extrínseca.

En el aprendizaje motor es fundamental que las personas reciban algún tipo de realimentación, ya sea de carácter extrínseco o intrínseco. 


\section{Manifestaciones de la realimentación extrínseca}

Cuando se brinda realimentación extrínseca (aumentada), esta se puede enfocar hacia el conocimiento del rendimiento de la acción motriz o hacia el conocimiento del resultado de esta última. La figura 2 expone las manifestaciones de la realimentación mencionadas.

Figura 2. Manifestaciones de la realimentación extrínseca

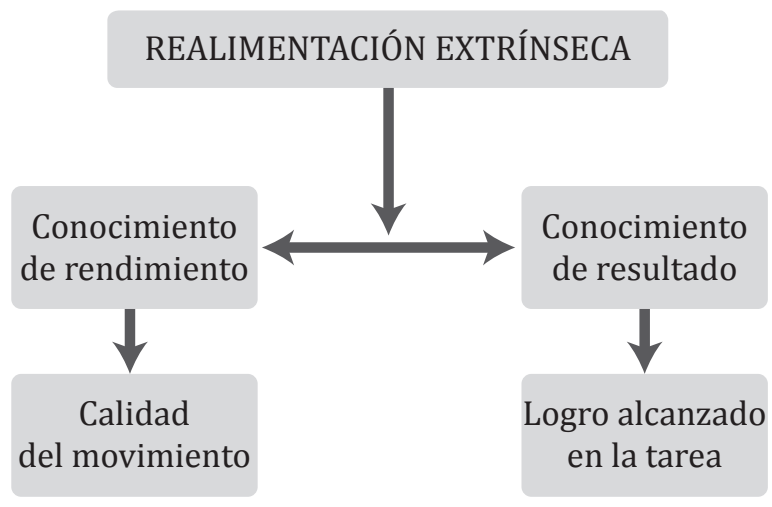

El conocimiento del rendimiento se refiere a la parte cualitativa del movimiento y nos brinda información acerca de la calidad del movimiento (Schmidt y Wrisberg, 2008; Schmidt, y Lee, 2005, y Zelaznik, 1996). Esta aproximación se ocupa de la realización de un especifico movimiento en términos de sus componentes como: rotación de la cadera en la habilidad de patear; acción de los brazos en la ejecución de un salto; ángulo de elevación de la rodilla al correr; ubicación del centro de gravedad con respecto a la base de sustentación en el patinaje; desplazamiento del peso del cuerpo sobre una u otra extremidad inferior; ubicación del objeto a proyectar en el aire con respecto a hombro del brazo que ejecuta la acción de lanzar; entre otras.

En términos generales, la realimentación extrínseca del conocimiento del rendimiento está dirigida hacia la corrección de patrones de movimiento inapropiados, mejor que hacia el resultado del movimiento. Estos aspectos, por lo general, son distintos de la realimentación intrínseca.

El conocimiento del resultado se refiere a la parte cuantitativa del movimiento y nos brindan información acerca del nivel de logro alcanzado en una tarea en particular (Schmidt y Wrisberg, 2008; Schmidt, y Lee,
2005; Zelaznik, 1996). Esta aproximación se enfoca en el producto de algunos movimientos específicos que el practicante realiza como: la distancia cubierta en un salto sin impulso previo; el tiempo empleado en correr una distancia determinada; la distancia alcanzada por algún objeto arrojado con una o dos manos; el número de pelotas impactadas con el bate en el deporte del béisbol; la cantidad de intentos realizados desde la línea de tiro libre en el deporte del baloncesto, y otras medidas del producto final del rendimiento de las habilidades motrices.

En términos generales, la realimentación extrínseca de conocimiento del resultado está dirigida hacia la corrección del resultado del movimiento en el ambiente. Estos aspectos frecuentemente son redundantes con la realimentación intrínseca.

\section{Consideraciones prácticas para brindar realimentación}

Las consideraciones prácticas para dar realimentación extrínseca que se mencionan a continuación son aspectos relevantes en todo proceso de enseñanza/ aprendizaje de habilidades motrices. Por lo general, la realimentación extrínseca que proporciona el profesor/ entrenador se realiza luego de la ejecución de una habilidad y se enuncia en forma verbal la mayoría de ocasiones (Fairbrother, 2010).

Los siguientes elementos contemplan implícitamente el uso de material tecnológico, como grabaciones en video, para apoyar el proceso de realimentación. Pero es necesario tener en cuenta, que una realimentación hecha con imágenes de video puede brindar demasiada información, especialmente si la habilidad es compleja y el practicante no sabe cuáles de los muchos detalles son importantes. Entonces, respecto a las consideraciones prácticas relevantes para dar realimentación, el profesor/entrenador bebe determinar algunos aspectos importantes como: qué realimentación brindar, cuán detallada debe ser, la frecuencia de proporcionar realimentación o el momento preciso de brindarla, entre otras (Wrisberg, 2007; Schmidt y Lee, 2005; y Schmidt y Wrisberg, 2008). Se retoma la propuesta de los autores mencionados, por ser quienes proponen de manera empírica consideraciones específicas para proporcionar realimentación. Aunque algunas de las siguientes consideraciones son complementadas con estudios aportados por otros autores. 


\section{Determinar el tipo de realimentación a brindar}

Cuando las personas tratan de aprender un programa motor, muchas veces tienen serios problemas, porque son incapaces de ajustar el programa de movimiento en la mejor forma posible. Por ejemplo, una jugadora de fútbol puede cabecear un balón caracterizado por un movimiento del tronco que inicia muy temprano, con respecto al inicio de la flexión del cuello. Con este ejemplo podemos identificar que un practicante puede hacer ajustes en la estructura temporal del movimiento o en los parámetros de este. Por ello, el practicante debe recibir realimentación de la estructura temporal del movimiento o recibir realimentación de sus parámetros.

Los ajustes en la estructura temporal del movimiento se refieren al acoplamiento o la diferenciación en la acción de los diferentes segmentos corporales. En el ejemplo de fútbol, la jugadora puede ser capaz de controlar el momento de iniciar el movimiento de la flexión del tronco, pero puede tener mayor dificultad en generar un patrón relativo a la estructura temporal entre los músculos del tronco y los músculos del cuello, porque estos movimientos ocurren casi simultáneamente.

Los ajustes en los parámetros del movimiento se refieren a aspectos de aplicación de velocidad, amplitud de movimientos y la proyección de fuerza en diferentes direcciones. Una vez el practicante ha alcanzado una coordinación global del programa motor deseado, puede empezar a practicar parámetros específicos que definen las características superficiales del movimiento, como duración, dirección y amplitud. Por ejemplo, el profesor/entrenador puede brindar realimentación de parámetros del movimiento, como: "cabecea el balón con menos fuerza", "cabecea el balón hacia tu lado derecho", para asistir a los practicantes en la selección de parámetros más efectivamente.

En etapas iniciales de aprendizaje de una nueva habilidad, es mejor que el profesor/entrenador brinde realimentación dirigida a la corrección de los errores en el patrón fundamental de movimiento o estructura temporal (Schmidt y Wrisberg, 2008). Aquí es importante que profesor/entrenador tenga claro que los procesos pueden ser un poco lentos, que la estructuración de un programa motor toma tiempo en adquirirse y que inefectivos en la estructura temporal son difíciles de eliminar.

Qué tan detallada debe ser la realimentación
Una consideración importante cuando se proporciona realimentación extrínseca es determinar cuán preciso o detallado debe ser esta. En etapas tempranas de aprendizaje, los errores del practicante son muy grandes, por lo que información detallada acerca de la exactitud de los errores no es importante. Investigaciones respecto a la precisión de la información sugirieron que la realimentación extrínseca no necesita ser extremadamente precisa para ser efectiva (Wrisberg, 2007).

Esto es particularmente verdadero cuando en etapas iniciales de aprendizaje, el practicante está tratando de obtener una idea general del programa motor correcto. En este momento, todo lo que el practicante necesita es información general acerca de la equivalencia de sus errores. Así, un profesor/entrenador puede decirle a un principiante en la prueba de salto alto, que su despegue fue un poco adelantado. Sin embargo, cuando el practicante alcanza un alto nivel de habilidad técnica, se podría beneficiar de una realimentación más precisa que le ayude a hacer ajustes más exactos en sus movimientos. Por ejemplo, al practicante de la prueba de salto alto se le debe dar información acerca del tiempo gastado, en centésimas de segundo, que empleó en dar los últimos dos pasos.

Núñez y Gálvez (2010) encontraron que la precisión de la realimentación de resultado, afecta negativamente el aprendizaje en un momento temprano del mismo; así como también, encontraron que el éxito obtenido a través de la provisión de una realimentación más precisa y cuantitativa tenía mayor efecto en las evaluaciones de retención del aprendizaje, que la realimentación menos precisa y cualitativa proporcionada a estudiantes universitarios de un programa en educación física.

\section{Determine la frecuencia para dar realimentación}

El profesor/entrenador debería considerar cómo y cuándo dar realimentación, en lugar de asumir que más realimentación es mejor. En otras palabras, la calidad de la realimentación brindada ha demostrado ser más importante que la cantidad; ya que una realimentación más frecuente, no es necesariamente mejor.

Intentos de prácticas seguidas de la ausencia de la realimentación son beneficiosos para el aprendizaje, pues el practicante es forzado a dedicar más atención a la información disponible intrínsecamente. Así, cuando a los practicantes se les da realimentación menos frecuente, ellos son menos susceptibles que se vuelvan 
dependientes de la realimentación. Por ejemplo, Perrota y Mele (2009) mencionan que una alta frecuencia de la realimentación de resultado durante el periodo de práctica interfiere con el aprendizaje; porque el practicante usa la información externa para guiar la respuesta y no es obligado a usar los procesos de memoria y buscar otras fuentes de información.

El profesor/entrenador debe decidir la frecuencia de dar la realimentación, con la idea de facilitar el aprendizaje motor en lugar de perjudicarlo. Como regla general, se debe dar realimentación más frecuente durante una etapa inicial de aprendizaje y progresivamente menos frecuente, en la medida en que los niveles de aprendizaje mejoren. Schmidt y Wrisberg (2008) mencionan que con deportistas experimentados - una vez son capaces de identificar la información relevante y producir efectivos movimientos-, ellos deberían tener poca necesidad de alimentación extrínseca. En algunas situaciones es necesario considerar la complejidad de la habilidad y la experiencia del practicante para determinar si dar realimentación o no.

\section{Cuál es el momento preciso para dar realimentación}

Los practicantes aprovechan más la realimentación cuando ellos preguntan por ella, que cuando alguien más decide que ellos la necesitan (Wrisberg, 2007). Los aprendices ponen más atención de cerca a sus sensaciones propioceptivas y exploran más la realimentación intrínseca disponible, cuando la realimentación extrínseca es demorada. Tal vez el instante preciso de dar realimentación no es inmediatamente luego de haber realizado la práctica de la habilidad, pues aquí es indispensable que el practicante explore más su realimentación inherente disponible (Anderson, Magill, Sekiya y Ryan, 2005).

La realimentación se debe demorar, no se debe dar inmediatamente el practicante ha realizado la ejecución. Hay alguna evidencia que sugiere que puede haber un detrimento en el aprendizaje si la realimentación se brinda inmediatamente luego de la práctica de una habilidad (Swinnen, Schmidt, Nicholson, y Shapiro, 1990). Debemos tener en cuenta que la realimentación que proporciona el profesor/entrenador, no debe interferir con el proceso intrínseco de la realimentación del practicante; pues una vez este es capaz de identificar la realimentación intrínseca relevante, necesitará menos realimentación extrínseca por parte del profesor/ entrenador.

Una buena estrategia es implementar el uso de palabras clave, como lo exponen Sanderson y Mc Clements, (1998), lo cual va a permitir darle tiempo al practicante para que interiorice sus sensaciones propioceptivas y, a su vez, poder sacar provecho del momento preciso para dar realimentación. Con el uso de palabras clave, profesor/entrenador y practicante pueden modificar una habilidad técnica, sobre todo si la habilidad practicada es cerrada; esta última es la que se realiza en un ambiente predecible o estacionario y le permite al deportista planear sus movimientos previamente a la ejecución (Schmidt y Wrisberg, 2008).

El uso de palabras clave para indicar que la ejecución se ha realizado correctamente es muy útil para permitir que el practicante internalice sus sensaciones propioceptivas de la habilidad ejecutada. Palabras clave como "bien" o "sí" pueden usarse para indicar una ejecución correcta; mientras que palabras como "mejor", pueden indicar un progreso, pero no el éxito total de la ejecución. Una palabra clave como "no" también es útil para indicar que no se ha logrado la ejecución deseada, ya que alerta al practicante a los efectos de evitar la internalización de las sensaciones propioceptivas de la habilidad ejecutada. Así, es importante que profesor/entrenador y practicante comprendan con exactitud el significado de cada palabra clave establecida, como lo mencionan Badets y Blandin (2005).

Es importante que el practicante disponga de tiempo para internalizar la sensación de la ejecución de la habilidad, sin interferencias externas. Por ello, el profesor/entrenador debe dejar solo al practicante por un momento, luego de que la palabra clave ha sido expresada y no intentar dar información técnica mientras el practicante está tratando de internalizar la ejecución correcta.

Un ejemplo de esto puede ser el de un deportista que intenta cambiar un elemento del servicio en voleibol. A través de conversaciones entre profesor/entrenador y practicante se ha acordado que la palabra clave "sí", indicará una ejecución correcta. También han acordado que cuando el mismo término es expresado, se permitirá que el atleta se tome un tiempo (alrededor de un minuto) para recordar la sensación de la ejecución. Luego, el 
atleta determina el momento en que está listo para recibir realimentación técnica y hablar de la ejecución con el entrenador (Sanderson y McClements, 1998).

\section{Cuánta información brindar}

En muchas ocasiones, los profesores/entrenadores están en posición de dar realimentación acerca de incontables características de los movimientos de los practicantes. Ellos tienen el potencial de sobrecargarlo de información y, como podremos suponer, esto puede ser un problema, pues el procesamiento de la información en el practicante es un tanto limitada.

En este sentido, la realimentación debe ser simple y directa. Una buena regla a tener en cuenta es considerar una sola característica del movimiento del practicante que sea fundamental (la más importante), enfocarse en ella y restringir la realimentación a esa característica para la mejora de la habilidad.

Además de trabajar la anterior estrategia, los profesores/entrenadores pueden implementar un método para evitar la sobrecarga de información en el alumno, proporcionando a los practicantes realimentación de resumen. La realimentación de resumen consiste en dar realimentación solamente después de un determinado número de intentos de la habilidad, digamos entre 5 a 15 (Schmidt, Lange y Young, 1990). Por ejemplo, un profesor de tenis que está ayudando a un practicante en el golpe de servicio cerca de la línea " $t$ ", puede solicitarle al practicante que golpee 10 servicios. Si el profesor registra en metros el lugar donde la bola cae con respecto a la línea, para cada uno de los 10 servicios y luego los grafica en una tabla; después del último intento del jugador, el profesor puede mostrarle los resultados y de esta forma proporcionar realimentación acerca de cada uno de los intentos.

El número de intentos que los profesores-entrenadores deberían tener en cuenta para brindar realimentación de resumen, probablemente depende de la complejidad de la habilidad a aprender. Para habilidades "simples", 12 ó 15 intentos pueden ser incluidos en la realimentación de resumen y para habilidades "complejas", cinco intentos serán suficientes a tener en cuenta (Wrisberg, 2007). Aunque, si la habilidad es muy exigente para el practicante, el número óptimo para brindar realimentación de resumen puede ser no más de uno o dos, al menos hasta que el practicante domine los elementos esenciales del movimiento. Sin embargo, Schmidt, Lange y Young, (1990), hallaron que la más efectiva realimentación de resumen se obtiene luego de cinco intentos.

Si el profesor/entrenador determina muy bien cuánta información dar, según la identificación de la característica más importante a corregir en el movimiento, $\mathrm{y}$ dependiendo del uso racional de la realimentación de resumen, los procesos de enseñanza/aprendizaje se pueden optimizar mejorando la efectividad de la realimentación y, a su vez, evitando los efectos de dependencia de la realimentación. Cuando se proporciona realimentación permanentemente, esta tiene la propiedad de que puede producir dependencia por parte del practicante; porque puede eliminar los efectos de la realimentación intrínseca y no permite aumentar el aprendizaje motor como lo evidencian Kilduski y Rice (2003).

Determine si debe cuestionar al practicante.

A veces es necesario ayudar al practicante desde la realimentación extrínseca en su proceso de realimentación intrínseca. De esta manera, explícitamente se le debe ayudar a identificar sus errores antes de dar la realimentación. Si se les pregunta a los practicantes que reflexionen sobre sus propios movimientos, antes de proporcionar la realimentación extrínseca, ellos pueden tener más sensibilidad con respecto a su información intrínseca (Schmidt y Lee, 2005). Por ejemplo, luego de un ejercicio de salto, en la prueba del salto largo, se le puede preguntar al atleta a qué altura ubicó la rodilla de la pierna libre en la fase de despegue o si tenía totalmente extendida la articulación de la rodilla de la pierna de rechazo en la misma fase. Luego, una vez el profesor/entrenador proporcione su realimentación, los practicantes podrán contrastar mejor su realimentación inherente con la realimentación extrínseca.

\section{Determine si la información debe ser verificada.}

En ocasiones, luego de brindar adecuadamente la realimentación es necesario que el profesor/entrenador verifique si el practicante entendió la información transmitida. Para estos casos, el profesor/entrenador debe solicitarle al practicante que en forma explícita, verbalice la información captada para confirmar si el mensaje de la realimentación está correcto y evitar que este sea mal interpretado o abandonado. Si el practicante ha entendido bien el mensaje, el profesor/ entrenador puede reafirmar la comunicación manifestando que eso era exactamente lo que quería decir. De 
otro lado, si el practicante ha entendido mal el mensaje, el profesor/entrenador debe aprovechar la ocasión para corregirlo y clarificarlo (Thompson, 2009).

\section{Consideraciones finales}

Cuando un deportista está en práctica de aprendizaje de una habilidad, requiere con frecuencia realimentación acerca de los ajustes que debe hacer para mejorar su desempeño. La información consignada en el presente documento indica que la realimentación extrínseca ha mostrado ser una herramienta poderosa que afecta positivamente el aprendizaje de habilidades (Marques, Ribeiro, Santos y Arroyo, 2010).

La calidad de la realimentación brindada ha demostrado ser más importante que la cantidad, como lo referencian Sadowski et al. (2011), quienes encuentran que en las primeras etapas de aprendizaje demasiada información verbal dificulta el proceso de adquisición de tareas complejas; o como menciona Claxton (1988), que compara la cantidad de realimentación empleada por un grupo de entrenadores de tenis con éxito y por un grupo de entrenadores con un menor éxito, y concluye que el primer grupo de entrenadores aporta menos realimentación que los entrenadores con menos éxito. En otras palabras, el profesor/entrenador deberá considerar cómo y cuándo dar realimentación, mejor que asumir que más realimentación es mejor. Sin embargo, los niños en edades prepuberales o en madurez temprana pueden necesitar más intentos de práctica con realimentación permanente, comparados con los adultos; esto con el propósito de formar una representación interna de la habilidad estable y precisa, como lo comentan Sullivan, Kantak y Burtner (2008).

\section{REFERENCIAS BIBLIOGRÁFICAS}

Anderson, D.; Magill, R.; Sekiya, H. y Ryan, G., (2005). Support for an explanation of the guidance effect in motor skill learning. Journal of Motor Behavior 37(3), 231-238.

Baechle, T. y Earle, R. (2008). Essentials of strength training and conditioning. National Strength Conditioning Association. 3a. ed. Champaign, EE.uU.: Human Kinetics.

Badets, A. y Blandin, Y. (2005). Observational learning: effects of bandwidth Knowledge of Results. Journal of Motor Behavior 37(3), 211-216.
En personas adultas el éxito obtenido a través de la provisión de una realimentación más precisa y cuantitativa, tiene mayor efecto en las evaluaciones de retención del aprendizaje, que la realimentación menos precisa y cualitativa, según los hallazgos de Núñez y Gálvez (2010).

Si el profesor entrenador determina en forma óptima, para un aprendiz en cuestión, la cantidad de realimentación a dar, evitará la creación de dependencia por parte del aprendiz hacia la realimentación, pues el exceso de realimentación puede ser una distracción que no permite aumentar el aprendizaje motor (Kilduski y Rice, 2003). Además, en algunos casos los profesores deben permitir a los aprendices tiempo para descubrir la relación entre ellos mismos, la tarea y el ambiente, antes de proporcionar realimentación extrínseca.

Cuando el ser humano practica y aprende una habilidad, nunca vuelve a ser la misma persona que era antes (Salmoni, Schmidt y Walter, 1984). Así, el aprendizaje tiene el efecto de cambiar al aprendiz (así sea ligeramente), en una relativa forma permanente. Pero, ¿qué tan permanente puede establecerse un cambio en la adquisición de programas motores?

Nuestra misión es clara: debemos dirigir los cambios en el aprendizaje motor con efectos perdurables y para ello una estrategia es optimizar los procesos de realimentación por medio de la aplicación de algunas consideraciones prácticas expuestas anteriormente. Aunque con sorpresa, en la actualidad podemos intuir que el vínculo entre las conceptualizaciones del aprendizaje motor y la práctica no ha sido aprovechado siempre en la forma que uno podría esperar.

Clark, M. y Russell, A. (2007). Essentials of performance enhancement. Calabasas, EE.UU.: National Academic Sport Medicine.

Claxton, D. (1988). A systematic observation of more and less successful high school tennis coaches. Journal of Teaching in Physical Education, 7, 302-310.

Davids, K.; Button, C. y Bennett, S. (2008). Dynamics of skill acquisition. Champaign, EE.UU.: Human Kinetics.

Fairbrother, J. (2010). Fundamentals of motor behavior. Champaign, EE.UU.: Human Kinetics. 
Geinisman, Y.; Berry, R.; Disterhoft, J.; Power, J. y Van der Zee, E. (2001). Associative Learning Elicits the Formation of Multiple-Synapse Boutons. The Journal of Neusoscience 21(15), 5568-5573.

Haywood, K. y Getchell, N. (2009). Life span motor development. 5a. ed. Champaign, EE.UU.: Human Kinetics.

Kilduski, N. y Rice, M. (2003). Qualitative and quantitative knowledge of results: Effects on motor learning. American Journal of Occupational Therapy 57(3), 329336.

Ma, L.; Narayana, S.; Robin, D.; Fox, P.y Xiong, J., (2011). Changes occur in resting state network of motor system during 4 weeks of motor skill learning. Neuroimage 58(1), 223-266.

Maguire, E. (2014). Memory consolidation in humans: New evidence and opportunities. Experimental Physiology 99(3), 471-486.

Marques, P.; Ribeiro, M.; Santos, G. y Arroyo, M. (2010). Análisis multidimensional del feedback pedagógico en entrenamiento en voleibol. Revista Internacional de Medicina y Ciencias de la Actividad Física y el Deporte 10(38), 181-202.

Marschall, F.; Bund, A. y Wiemeyer, J. (2007). Does Frecuent Augmented Feedback Really degrade Learning? A Meta-Analysis. E-journal Bewegung und Training, 1, 74-85.

Martin, D. et al. (2004). Metodología general del entrenamiento infantil y juvenil. Barcelona, España: Paidotribo.

Núñez, F. y Gálvez, J. (2010). Influence of three accuracy levels of knowledge of results on motor skill acquisition. Journal of Human Sport and Performance 5(3), 476-484.

Perrota, F. y Mele, E. (2009). The motor learning, memory, knowledge of results. Comparative analysis of homogeneous groups. Journal of Physical Education and Sports 25(4), 1-9.

Sadowski, J.; Mastalerz, A.; Niznikowski, T.; Wisniowski, W.; Biegajlo, M. y Kulik, M. (2011). The effects of different types of verbal feedback on learning a complex movement task. Polish Journal of Sport and Tourism 18(4), 308-310.
Salmoni, A.; Schmidt, R. y Walter, C, (1984). Knowledge of Results and Motor Learning: A Review and Critical Reappraisal. Psychological Bulletin 95(3), 355-386.

Sanderson, L. y McClements, J. (1998). A coach's perspective on skill learning. New Studies in Athletics 13(3), 33-38.

Schmidt, R. (2003). Motor Schema Theory After 27 Years: Reflections and Implications for a New Theory. Research Quarterly for Exercise and Sport 74(4), 336375.

Schmidt, R.4a. ed. Champaign, EE.UU.: Human Kinetics.

Schmidt, R.Motor Learning and Performance.4a. ed. Champaign, EE.UU.: Human Kinetics.

Schmidt, R.; Lange, C. y Young, D. (1990). Optimizing Summary Knowledge of Results for Skill Learning. Human Movement Science, 9, 325-348.

Schmidt, R., y Lee, T. (2005). Motor control and learning. A behavioral Emphasis. Fourth Edition. Champaign, United States: Human Kinetics.

Schmidt, R., y Wrisberg, C. (2008). A Situation-Based Learning Approach. Shea, C. y Wulf, G. (2005). Schema theory: A critical appraisal and reevaluation. Journal of Motor Behavior 37(2), 85-101.

Sullivan, K.; Kantak, S. y Burtner, P. (2008). Motor learning in children: Feedback effects on skill acquisition. Physical Therapy, 88, 720-732.

Swinnen, S.; Schmidt, R.; Nicholson, D. y Shapiro, D., (1990). Information feedback for skill acquisition: Instantaneous knowledge of results degrades learning. Journal of Experimental Psychology: Learning, Memory and Cognition, 16, 706-716.

Thompson, P. (2009). Introduction to Coaching. The Official IAAF Guide to Coaching Athletics. Lincolnshire, Inglaterra: Descartes Publishing.

Wrisberg, C. (2007). Sport skill instruction for coaches. Champaign, EE.UU.: Human Kinetics.

Wilmore, J. y Costill, D. (2002). Fisiología del esfuerzo y del deporte. 5a. ed. Barcelona, España: Paidotribo.

Zelaznik, H. (1996). Advances in motor learning and control. Champaign, EE.UU.: Human Kinetics. 